\title{
ON THE DIFFERENTIABILITY OF GENERALIZED SOLUTIONS OF FIRST ORDER ELLIPTIC EQUATIONS WITH DISCONTINUOUS COEFFICIENTS $\left({ }^{1}\right)$
}

\author{
BY \\ ALFRED SCHATZ
}

1. Introduction. In this paper we shall consider uniformly elliptic equations in the complex plane of the form

$$
w_{\bar{z}}=A(z) w_{z}+B(z) w+C(z) \bar{w}+D(z) .
$$

In particular our chief concern will be with the differentiability properties of generalized solutions of (1.1) in the neighborhood of a point on an arc across which the coefficients of (1.1) have finite jump discontinuities.

A well-known local result [1], [5] may be stated as follows: If the coefficients of (1.1) are Hölder continuous in $\bar{G}$, the closure of a disk $G$ with center at a point $t_{0}$, then there exists a closed disk $\bar{G}_{1}$ with center at $t_{0}$ and $\bar{G}_{1} C G$ such that the first derivatives of any generalized solution $\left({ }^{2}\right) w(z)$ of $(1.1)$ in $G$ are Hölder continuous in $\bar{G}_{1}$. Suppose now that $G$ is separated into two parts $G^{+}$and $G^{-}$by a "sufficiently smooth" simple arc $L$ passing through $t_{0}$, and that the coefficients $A(z), B(z)$, $C(z)$, and $D(z)$ are Hölder continuous in each of the closed regions $\bar{G}^{+}$and $\bar{G}^{-}$ and therefore have at most finite jump discontinuities across $L$. In Theorem 1 , it is shown that there exists a suitably chosen disk $G_{0}$ with center at $t_{0}$ and $\bar{G}_{0} C G$, such that the first derivatives $w_{z}$ and $w_{\bar{z}}$ of any generalized solution of (1.1) are Hölder continuous in each of the two closed regions $\bar{G}_{0}^{+}$and $\bar{G}_{0}^{-}$separately. This says that the derivatives $w_{z}$ and $w_{\bar{z}}$ are smooth up to $L$ in $\bar{G}_{0}$ from either side of $L$ and have at most finite jump discontinuities across it.

In $\S 4$ we specialize (1.1) to Beltrami’s equation

$$
w_{z}=A(z) w_{z} \text {. }
$$

Many problems in analysis and geometry may be reduced to the problem of reducing positive differential quadratic forms [1], with coefficients which have discontinuities of the first kind across contours, to a canonical form. The above problem in turn can be reduced to proving the existence of homeomorphic solutions of (1.2). In Theorem 2 it is shown that if $A(z)$ satisfies the conditions previously

Received by the editors August 26, 1965.

(1) This paper was supported by Office of Naval Research Contract Nonr(G)00028-64.

$\left({ }^{2}\right)$ That is, $w(z)$ has generalized first derivatives in the Sobolev sense which belong to $L_{p}^{\text {loc }}(G)$ for some $p>2$ and satisfy (1.1) almost everywhere in $G$. See also $\$ 2$ and 3. 
imposed then every homeomorphic generalized solution of (1.2) has in addition to the properties stated in Theorem 1, a nonvanishing Jacobian in each of the closed regions $\bar{G}_{0}^{+}$and $\bar{G}_{0}^{-}$and that the image of $\bar{L}_{0}$, where $\bar{L}_{0}=\bar{G}_{0} \cap L$, is also a smooth arc. This result may also be used to solve Riemann problems with shifts on smooth contours by reducing them to Riemann problems without shifts.

In $\S 5$, the results of Theorems 1 and 2 are extended to generalized solutions of quasilinear uniformly elliptic equations of the form

$$
w_{z}=a(z, w) w_{z}+b(z, w),
$$

under suitable smoothness conditions on the coefficients.

The proofs of the results in Theorems 1 and 2 are modifications of a method which is used to prove differentiability properties for solutions of (1.1) in the case that the coefficients are Hölder continuous. It is based on a representation Theorem due to Morrey [3], and Bers and Nirenberg [4], for generalized solutions of (1.1) in which the coefficients can be bounded measurable functions. The representation theorem says that every solution of (1.1) may be written in terms of analytic functions of special solutions of (1.1) and thus the proofs of Theorems 1 and 2 are reduced to that of finding special solutions with the required regularity properties. This is done in $\S 3$. The results for (1.3) follow easily from Theorems 1 and 2.

I would like to express my thanks to Professor $\mathrm{H}$. Weitzner for having suggested a problem which led to this investigation, Professor $L$. Bers for his valuable advice and Miss G. Wollmer for her encouragement during this work.

2. Notation, norms, and generalized derivatives. Let $S$ be a bounded open region in the complex $z=x+i y$ plane, $S$ its boundary and $\bar{S}$ the closure of $S$. Let $f(z)$ be a function defined on $S$. By $f_{z}$ and $f_{\tilde{z}}$ we mean the formal derivatives

$$
\frac{\partial f}{\partial z} \equiv f_{z} \equiv \frac{1}{2}\left(f_{x}-i f_{y}\right), \quad \frac{\partial f}{\partial \bar{z}} \equiv f_{\bar{z}} \equiv \frac{1}{2}\left(f_{x}+i f_{y}\right) .
$$

We shall often consider functions $f(z)$ which are at first defined in $S$ and then speak of the values of $f(z)$ (and possibly $f_{z}$ and $f_{z}$ ) in $\bar{S}$. By this we mean that $f(z)$ (and possibly $f_{z}$ and $f_{z}$ ) have limits from $S$ at every point $z_{0}$ on $\dot{S}$ and these are to be taken as the values of the function on $\dot{S}$.

A function $f(z)$ is said to satisfy a Hölder condition on $\bar{S}$ with exponent $0<\mu \leqq 1$ if

$$
H(f, \bar{S}, \mu)=\operatorname{liub}_{z_{1}, z_{2} \in S: z_{1} \neq z_{2}} \frac{\left|f\left(z_{1}\right)-f\left(z_{2}\right)\right|}{\left|z_{1}-z_{2}\right|^{\mu}}<\infty .
$$

We now introduce the following Banach spaces for functions defined on $\bar{S}$.

$C(\bar{S})$ : The space of continuous functions with norm

$$
C(f, \bar{S})=\max _{z \in S}|f(z)| .
$$


$C_{\mu}(\bar{S}):$ The space of functions satisfying

$$
C_{\mu}(f, \bar{S})=C(f, \bar{S})+H(f, \bar{S}, \mu)<\infty \quad \text { for } 0<\mu<1 .
$$

$\operatorname{Lip}(f, \bar{S}): \quad$ The space of functions satisfying

$$
\operatorname{Lip}(f, \bar{S})=C(f, \bar{S})+H(f, \bar{S}, 1)<\infty .
$$

$C_{\mu}^{1}(\bar{S})$ : The space of functions having continuous first derivatives in $\bar{S}$ and

$$
C_{\mu}^{1}(f, \bar{S})=C(f, \bar{S})+C\left(f_{z}, \bar{S}\right)+C\left(f_{\bar{z}}, \bar{S}\right)+H\left(f_{z}, \bar{S}, \mu\right)+H\left(f_{\bar{z}}, \bar{S}, \mu\right)<\infty .
$$

$L_{p}^{\text {loc }}(S)$ : The set of functions defined on $S$ (not a Banach space), which are summable to the $p$ th power $p \geqq 1$ on every compact $S_{1}$ of $S$ and

$$
\left(\iint_{S_{1}}|f(z)|^{p} d z d \bar{z}\right)^{1 / p}<M_{S_{1}}
$$

$L_{p}(S): \quad$ The space of functions which are summable to the $p$ th power and

$$
L_{p}(f, S)=\left(\iint_{S}|f|^{p} d z d \bar{z}\right)^{1 / p}<\infty
$$

Let $L$ be a simple rectifiable arc which contains its endpoints. $L$ is said to belong to $C_{\mu}^{1}$ for some $0<\mu \leqq 1$, if when it is written in parametric form with respect to arc length $s$

$$
z(s)=x(s)+i y(s)
$$

then the function $z(s)$ has a first derivative which satisfies a Hölder condition with exponent $\mu$ for $0<s \leqq l$, where $l$ is the length of the arc.

We shall now consider the concept of generalized derivatives in the Sobolev sense and in particular restrict ourselves to the complex plane. For an account of this theory which is more than sufficient for the purposes of this paper the reader is referred to Vekua [1].

Denote by $D_{1}^{0}(S)$ the class of functions having compact support in $S$ and continuous first derivatives in $S$.

Definition. If $f, g \in L_{1}^{\text {loc }}(S)$ and satisfy the relation

$$
\begin{gathered}
\iint_{S} f \frac{\partial \phi}{\partial \bar{z}} d z d \bar{z}+\iint g \phi d z d \bar{z}=0 \\
\left(\iint_{S} f \frac{\partial \phi}{\partial z} d z d \bar{z}+\iint g \phi d z d \bar{z}=0\right),
\end{gathered}
$$

where $\phi$ is an arbitrary function of class $D_{1}^{0}(S)$, then $g$ is said to be the generalized derivative of $f$ with respect to $\bar{z}$ (with respect to $z$ ). 
We remark that if $g \in L_{p}^{\text {loc }}(S)$ for some $p>2$ and if $g$ is the generalized derivative of a function $f \in L_{1}^{\text {loc }}(G)$, then

$$
f \in C_{\rho}\left(S_{1}\right)
$$

where $\rho=(p-2) / p$ and $S_{1}$ is any compact subset of $S$.

3.1. Statement of Problem and Results. Let $A(z), B(z), C(z)$, and $D(z)$ be bounded measurable functions defined in a region $S$. By a generalized solution (or just solution) $w(z)$ of

$$
w_{\bar{z}}=A(z) w_{z}+B(z) w+C(z) \bar{w}+D(z),
$$

we mean any function $w(z)$ defined in $S$, which has generalized first partial derivatives in the Sobolev sense belonging to $L_{p}^{\mathrm{loc}}(S)$ for some $p>2$, and which satisfies (3.1) almost everywhere in $S$.

Let $G$ be an open disk of radius $R$ with center at $t_{0}$, and denote by $G$ its boundary. Let $L$ be a simple arc passing through $t_{0}$ which intersects $G$ at two points and separates $G$ into two regions $G^{+}$and $G^{-}$. We shall make the following assumptions regarding $L, A(z), B(z), C(z)$, and $D(z)$ which will be called

CoNDitions I.

(i) $A(z), B(z), C(z), D(z) \in C_{\mu}\left(\bar{G}^{ \pm}\right)$for some $0<\mu<1$.

(ii) $|A(z)| \leqq K<1$ in $\bar{G}^{ \pm}$for some constant $K$.

(iii) $L \in C_{\mu}^{1}, \mu$ as in (i).

We remark that (ii) says that (3.1) is uniformly elliptic and that (i) says that the coefficients of (3.1) satisfy Hölder conditions in the closure of the regions $G^{+}$ and $G^{-}$separately, but are allowed to be discontinuous across $L$. In what follows, we shall show that under Conditions I, the first derivatives of any solution have this same property in an appropriately taken neighborhood of $t_{0}$ which is contained in $G$. The result to be proved is:

THEOREM 1. Under the hypothesis of Conditions I, there exists an open disk $G_{0}$ about $t_{0}, \bar{G}_{0} C G$, such that every solution $w(z)$ of (3.1) has the properties

$$
w_{z}, w_{\bar{z}} \in C_{\mu}\left(\bar{G}_{0}^{ \pm}\right)
$$

and therefore

$$
w(z) \in \operatorname{Lip}\left(\bar{G}_{0}\right),
$$

where $G_{0}^{+}$and $G_{0}^{-}$denote the intersections of $G_{0}$ with $G^{+}$and $G^{-}$respectively and $\mu$ is given in Conditions $\mathrm{I}$.

REMARK. Since the properties sought for are local in nature, we shall assume that $R$ is sufficiently small so that any circle of radius $r \leqq R$ about $t_{0}$ intersects $L$ 
at exactly two points and separates the disk of radius $r$ into two regions. This can always be done since $L \in C_{\mu}^{1}, 0<\mu<1$.

3.2. Preliminary to the Construction of Special Solutions. For the purpose of proving Theorem 1 it will be sufficient for us to consider the inhomogeneous Beltrami equation.

$$
w_{\bar{z}}=A(z) w_{z}+F(z),
$$

where $F(z)$ satisfies (i) of Conditions I. Thus the problem reduces to that of constructing the particular solutions of (3.4) with the properties we desire. As a first step in the construction of these solutions, we introduce a change of independent variables so that the region $G$ is transformed into one in which $L$ is mapped onto a straight line segment. To accomplish this, we shall assume that $R$ satisfies the conditions of the following lemma.

LEMMA 1. For $R$ sufficiently small, there exists an orientation preserving homeomorphism $\zeta=\psi_{1}(z)$ of $\bar{G}$ onto another closed region $\bar{G}_{1}$ such that:

(i) $L$ is mapped onto a segment $L_{1}$ of the real axis.

(ii) $\bar{G}_{1}^{ \pm}$, the images of $\bar{G}^{ \pm}$, lie in the closed upper half and closed lower half planes respectively.

(iii) Denote by $z=\chi_{1}(\zeta)$ the inverse mapping, then $\psi_{1}(z) \in C_{\mu}^{1}(\bar{G}), \chi_{1}(\zeta) \in C_{\mu}^{1}\left(\bar{G}_{1}\right)$ and $\left|\zeta_{z}\right|^{2}-\left|\zeta_{\bar{z}}\right|^{2} \equiv 1$ in $\bar{G}$.

(iv) $\zeta_{\bar{z}}=\beta(z) \zeta_{z}$ in $G$, where $\beta(z)$ is a known function having the properties $|\beta(z)| \leqq$ $\beta_{0}<1$ in $\bar{G}$ and $\beta(z) \in C_{\mu}(\bar{G})$.

The proof of Lemma 1 follows from the properties of $L$ and the implicit function theorem. We shall not give the details here.

Assuming for the present that $w(z)$ has continuous derivatives in $G^{ \pm}$, we have that $w$ satisfies the differential equation

$$
w_{\bar{\zeta}}=A_{1}(\zeta) w_{\zeta}+F_{1}(\zeta)
$$

where

$$
A_{1}(\zeta)=\frac{A(z)-\beta(z)}{1-A(z) \overline{\beta(z)}} \cdot \frac{\zeta_{z}}{\bar{\zeta}_{\bar{z}}}, \quad F_{1}(\zeta)=F\left(\chi_{1}(\zeta)\right),
$$

and hence from Lemma 1,

$$
A_{1}(\zeta), F_{1}(\zeta) \in C_{\mu}\left(\bar{G}_{1}^{ \pm}\right), \quad\left|A_{1}(\zeta)\right| \leqq K_{1}<1 \text { in } \bar{G}_{1}
$$

for some constant $K_{1}$.

Define the transformation $\eta=\psi_{2}(\zeta)$ as follows

$$
\eta=\psi_{2}(\zeta)=\frac{\zeta+A_{1}^{ \pm}(0) \zeta}{1+A_{1}^{ \pm}(0)}
$$

where the + sign is taken for $\operatorname{Im} \zeta>0$ and the - sign for $\operatorname{Im} \zeta \leqq 0$. 
As defined $\psi_{2}(\zeta)$ is a sectionally affine transformation which maps the $\zeta$ plane homeomorphically onto the $\eta$ plane, sending the u.h.p. and the 1.h.p. onto the u.h.p. and 1.h.p. respectively and leaves the real axis fixed. Denote by $\zeta=\chi_{2}(\eta)$ the inverse mapping. Then it follows from (3.7), that

$$
\zeta=\chi_{2}(\eta)=\frac{\left(1+A_{1}^{ \pm}(0)\right) \eta-A_{1}^{ \pm}(0)\left(1-A_{1}^{ \pm}(0)\right) \bar{\eta}}{1-\left|A_{1}^{ \pm}(0)\right|^{2}}
$$

where the + sign is taken for $\operatorname{Im} \eta \geqq 0$ and the minus sign for $\operatorname{Im} \eta<0$.

Hence $\psi_{2}(\zeta)$ and $\chi_{2}(\eta)$ have first derivative with respect to $\zeta, \zeta$, and $\eta, \bar{\eta}$ respectively which are constants and in general are discontinuous across the real axis unless $A_{1}^{+}(0)=A_{1}^{-}(0)$.

Denote by $G_{2}, G_{2}^{+}, G_{2}^{-}, L_{2}$, etc., the images of $G_{1}, G_{1}^{+}, G_{1}^{-}, L$, etc., under the mapping $\eta=\psi_{2}(\zeta)$. Introducing $\eta$ as a new independent variable, we find that $w$ satisfies the differential equation

$$
w_{\bar{\eta}}=A_{2}(\eta) w_{\eta}+F_{2}(\eta) \text { in } G_{2}^{ \pm},
$$

where

$$
A_{2}(\eta)=A_{2}^{ \pm}(\eta)=\frac{A_{1}^{ \pm}(\zeta)-A_{1}^{ \pm}(0)}{1-A_{1}^{ \pm}(\zeta) \overline{A_{1}^{ \pm}(0)}} \frac{\overline{1+A_{1}^{ \pm}(0)}}{1+A_{1}^{ \pm}(0)} \quad \text { for } \eta \in G_{2}^{ \pm}
$$

and where it is easily verified that

$$
A_{2}(\eta), F_{2}(\eta) \in C_{\mu}\left(\bar{G}_{2}^{ \pm}\right)
$$

Furthermore, the following properties of $A_{2}(\eta)$ follow from (3.6), (3.9), and (3.10):

$$
A_{2}^{ \pm}(0)=0
$$

There exist constants $K_{2}$ and $M$ such that

$$
\begin{array}{rr}
\left|A_{2}^{ \pm}(\eta)\right| \leqq K_{2}<1, & \eta \in G_{2}^{ \pm}, \\
\left|A_{2}\left(\eta_{1}\right)-A_{2}\left(\eta_{2}\right)\right| \leqq M\left|\eta_{1}-\eta_{2}\right|^{\mu}, & \eta_{1}, \eta_{2} \in G_{2}^{ \pm} .
\end{array}
$$

It follows directly from (3.12) and (3.13) that

$$
\left|A_{2}^{ \pm}(\eta)\right| \leqq M|\eta|^{\mu}, \quad \eta \in \bar{G}_{2}^{ \pm} .
$$

Since the origin is an interior point of $G_{2}$, there exists a disk $G_{\delta}$ of radius $\delta$ such that $\bar{G}_{\delta} C G_{2}$. Obviously (3.9) through (3.14) hold in $\bar{G}_{\delta}^{ \pm}$(the intersections of $\bar{G}_{2}^{ \pm}$with $\bar{G}_{\delta}$ respectively). 
Consider the function $A_{\delta}(\eta)$ defined by

$$
\begin{aligned}
A_{\delta}(\eta)=A_{\delta}^{ \pm}(\eta) & =A_{2}^{ \pm}(\eta) & & \text { for }|\eta|<\delta / 2, \eta \in \bar{G}_{\delta}^{ \pm}, \\
& =2 A_{2}^{ \pm}(\eta)(1-|\eta| / \delta) & & \text { for } \delta / 2 \leqq|\eta| \leqq \delta, \eta \in G_{\delta}^{ \pm}, \\
& =0 & & \text { for }|\eta|>\delta .
\end{aligned}
$$

Using (3.13), (3.14), and (3.15), it can be shown that

$$
\begin{array}{cl}
\left|A_{\delta}^{ \pm}(\eta)\right| \leqq M|\eta|^{\mu} & \text { for } \eta \in \bar{G}_{\delta}^{ \pm}, \\
\left|A_{\delta}^{ \pm}\left(\eta_{1}\right)-A_{\delta}^{ \pm}\left(\eta_{2}\right)\right| \leqq 5 M\left|\eta_{1}-\eta_{2}\right|^{\mu} & \text { for } \eta_{1}, \eta_{2} \in \bar{G}_{\delta}^{ \pm} .
\end{array}
$$

Let

$$
0<\delta<1
$$

then

$$
C\left(A_{\delta}, \bar{G}_{\delta}^{ \pm}\right) \leqq M \delta^{\mu}, \quad C_{\mu}\left(A_{\delta}, \bar{G}_{\delta}^{ \pm}\right) \leqq 5 M+M \delta^{\mu} \leqq 6 M .
$$

In a similar fashion, consider the function $F_{\delta}(\eta)$ defined by

$$
\begin{aligned}
F_{\delta}(\eta) & =F_{\delta}^{ \pm}(\eta)=F_{2}^{ \pm}(\eta) & & \text { for }|\eta| \leqq \delta / 2, \eta \in \bar{G}_{\delta}^{ \pm}, \\
& =2 F_{2}^{ \pm}(\eta)(1-|\eta| / \delta) & & \text { for } \delta / 2<|\eta| \leqq \delta, \eta \in \bar{G}_{\delta}^{ \pm}, \\
& =0 & & \text { for }|\eta|>\delta .
\end{aligned}
$$

It follows from (3.19) that $F_{\delta} \in C_{\mu}\left(\bar{G}_{\delta}^{ \pm}\right)$and

$$
C\left(F_{\delta} \bar{G}_{\delta}^{ \pm}\right) \leqq C\left(F_{2}, \bar{G}_{\delta}^{ \pm}\right), H\left(F_{\delta}, \bar{G}_{\delta}^{ \pm}, \mu\right) \leqq 5 H\left(F_{2}, \bar{G}_{\delta}^{ \pm}, \mu\right)+2 \delta^{-\mu} C\left(F_{2}, \bar{G}_{\delta}^{ \pm}\right),
$$

and hence

$$
C_{\mu}\left(F_{\delta}, \bar{G}_{\delta}^{ \pm}\right) \leqq 5 \delta^{-\mu} C_{\mu}\left(F_{2}, \bar{G}_{\delta}^{ \pm}\right)
$$

Instead of the differential equation (3.9), we shall investigate the differential equation

$$
U_{\bar{\eta}}=A_{\delta}(\eta) U_{\eta}+F_{\delta}(\eta), \quad \eta \in E,
$$

where $E$ is the finite $\eta$ plane and the functions $A_{\delta}(\eta)$ and $F_{\delta}(\eta)$ are given by (3.15) and (3.19).

Along with (3.21), we shall investigate the Beltrami equation

$$
V_{\bar{\eta}}=A_{\delta}(\eta) V_{\eta}, \quad \eta \in E .
$$

Our aim is to construct two functions $V(\eta)$ and $U(\eta)$, the first being a homeomorphic solution of (3.22) and the second a solution of (3.21), both having the 
property that their first order derivatives are Hölder continuous in $\bar{G}_{\delta}^{ \pm}$. From the fact that $A_{\delta}(\eta)=A_{2}(\eta), F_{\delta}(\eta)=F_{2}(\eta)$ in $G_{\delta / 2}$, it will follow on transforming these functions back to the $z$ plane, that they are respectively a homeomorphic solution of $V_{z}=A(z) V_{z}$ and a solution of $U_{\bar{z}}=A(z) U_{z}+F(z)$ in some neighborhood $G_{3}$ of $t_{0}$, and they have Hölder continuous derivatives in $\bar{G}_{3}^{ \pm}$.

We shall first consider the following Banach space. Let $L_{\delta}$ be the segment of the real axis $-\delta \leqq \eta_{1} \leqq \delta$, where $\eta=\eta_{1}+i \eta_{2}$. Denote by $B$ the set of functions $f(\eta)$ having the following properties:

(a) $f(\eta)$ is defined and continuous everywhere on $E-L_{\delta}$ (where $E$ denotes the finite $\eta$ plane).

(b) $f(\eta)$ vanishes outside of $G_{\delta} U L_{\delta}$.

(c) $f(\eta) \in C_{\mu}\left(\bar{G}_{\delta}^{ \pm}\right), 0<\mu<1$.

(d) Let $f^{ \pm}(\eta)$ denote the values of $f(\eta)$ in the u.h.p. and 1.h.p., respectively, then $f^{ \pm}(0)=0$.

It is easily verified that $B$ is a Banach space under the norm

$$
\|f\|_{B}=C_{\mu}\left(f, \bar{G}_{\delta}^{+}\right)+C_{\mu}\left(f, \bar{G}_{\delta}^{-}\right) .
$$

It follows from (c) and (d) that for any $f \in B$

$$
\left|f^{ \pm}(\eta)\right| \leqq H\left(f, \bar{G}_{\delta}^{ \pm}, \mu\right)|\eta|^{\mu} .
$$

We remark that it follows from (3.12), (3.15), (3.18), and (3.19) that $A_{\delta} \in B$. In fact, let $h(\eta)$ be any function which is continuous on $E-L_{\delta}$ and such that $h(\eta) \in C_{\mu}\left(\bar{G}_{\delta}^{ \pm}\right)$. Then,

$$
A_{\delta}(\eta) h(\eta) \in B
$$

3.3. Beltrami's Equation, Construction of a Local Homeomorphism. We shall first consider the Beltrami equation (3.22), that is

$$
V_{\bar{\eta}}=A_{\delta}(\eta) V_{\eta} \text { in } \quad E .
$$

Following the main steps used by Lichtenstein [7], [1] in constructing homeomorphic solutions of Beltrami's equation with Hölder continuous coefficients, we shall construct a homeomorphic solution of (3.26). The difference here is the use of the Banach space $B$. We look for a solution of the form

$$
V(\eta)=\eta-\frac{1}{\pi} \iint_{E} \frac{f(z) d z d \bar{z}}{z-\eta}=\eta-\frac{1}{\pi} \iint_{G_{0}} \frac{f(z) d z d \bar{z}}{z-\eta}=\eta+T(f),
$$

where $f \in B$.

For $\eta \in E-L_{\delta}$ we have

$$
V_{\bar{\eta}}=f(\eta), \quad V_{\eta}=1+S(f)=1-\frac{1}{\pi} \iint_{G_{\delta}} \frac{f(z) d z d \bar{z}}{(z-\eta)^{2}},
$$


where the last integral is to be taken in the principal value sense. Substituting (3.28) into (3.27), we obtain

$$
f(\eta)-A_{\delta}(\eta) S(f)=A_{\delta}(\eta)
$$

which is an integral equation for $f \in B$.

It will be shown that $S(f)$ is a contractive mapping of $B$ into itself provided $\delta$ is taken sufficiently small and hence since $A_{\delta}(\eta) \in B$, there will exist a unique solution $f \in B$ of (3.29). The proof that $S(f)$ is contraction mapping is quite lengthy. We shall need the following two lemmas. The proof of Lemma 2 follows easily from well-known properties of singular integrals and will not be included. The proof of Lemma 3 will be left for the appendix.

LEMMA 2. Let $f \in B$, then $S(f)$ is continuous at all points $\eta \in E-L_{\delta}$.

LEMMA 3. Let $f \in B$ then $S(f) \in C_{\mu}\left(\bar{G}_{\delta}^{ \pm}\right)$and

$$
\begin{gathered}
C\left(S(f), \bar{G}_{\delta}^{ \pm}\right) \leqq \frac{10 \delta^{\mu}}{\mu}\|f\|_{B}, \\
H\left(S(f), \bar{G}_{\delta}^{ \pm}, \mu\right) \leqq\left(2 M_{\mu}^{\prime}+25\right)\|f\|_{B},
\end{gathered}
$$

and hence

$$
C_{\mu}\left(S(f), \bar{G}_{\delta}^{ \pm}\right) \leqq\left(10 / \mu+2 M_{\mu}^{\prime}+25\right)\|f\|_{B},
$$

where $M_{\mu}^{\prime}$ is a constant depending only on $\mu$ and where $0<\delta<1$.

It follows from Lemma 2 and Lemma 3 that for any $f \in B, S(f)$ is continuous outside $L_{\delta}$ and $S(f) \in C_{\mu}\left(\bar{G}_{\delta}^{ \pm}\right)$. Hence, using (3.25), we have that

$$
A_{\delta} S(f) \in B,
$$

that is $A_{\delta} S(f)$ maps $B$ into itself. Since

$$
C_{\mu}\left(A_{\delta} S(f), \bar{G}_{\delta}^{ \pm}\right) \leqq C\left(A_{\delta}, \bar{G}_{\delta}^{ \pm}\right) C_{\mu}\left(S(f), \bar{G}_{\delta}^{ \pm}\right)+C_{\mu}\left(A_{\delta}, \bar{G}_{\delta}^{ \pm}\right) C\left(S(f), \bar{G}_{\delta}^{ \pm}\right)
$$

we have using (3.18), (3.23), (3.30), and (3.32) in (3.34)

$$
\left\|A_{\delta} S(f)\right\|_{B} \leqq 2 M\left(70 / \mu+2 M_{\mu}^{\prime}+25\right) \delta^{\mu}\|f\|_{B}=M_{\mu}^{\prime \prime} \delta^{\mu}\|f\|_{B},
$$

where $M_{\mu}^{\prime \prime}=2 M\left(70 / \mu+2 M_{\mu}+25\right)$ and both $M$ and $M_{\mu}^{\prime}$ and hence $M_{\mu}^{\prime \prime}$ are independent of $\delta$.

Choose $\delta$ so that

$$
0<\left(\frac{120 M+\mu M_{\mu}^{\prime \prime}}{\mu}\right) \delta^{\mu}<1, \quad 0<\delta<1,
$$


then

$$
M_{\mu}^{\prime \prime} \delta^{\mu}<1
$$

Hence, by a well-known theorem on contraction mappings [8] it follows that (3.29) has a unique solution $f \in B$. Thus the function $V$ defined by (3.27) is a solution of (3.29) and from (3.28) and Lemma 3 we have that

$$
V_{\eta}, V_{\bar{\eta}} \in C_{\mu}\left(\bar{G}_{\delta}^{ \pm}\right) \text {. }
$$

It follows from (3.27) that $V(z)$ is continuous in the plane and from (3.38) that $V \in \operatorname{Lip}\left(\bar{G}_{\delta}\right)$.

Therefore, $V(\eta)$ satisfies a Lipschitz condition in $\bar{G}_{\delta}$ and has Hölder continuous derivatives in $\bar{G}_{\delta}^{+}$and $\bar{G}_{\delta}^{-}$separately but may have jump discontinuities across $L_{\delta}$. Since $A_{\delta}(\eta)=0$ outside $\bar{G}_{\delta}$ and since the solution of $f \in B$ of the integral equation (3.29) belongs to $L_{p}(E)$ for any $1 \leqq P<\infty$, it follows from a theorem of Bojarski [9], [1] that $V(\eta)=\eta+T(f)$ establishes a homeomorphic mapping of the $\eta$ plane onto the $V$ plane. Returning now to the independent variable $z$, we see from (3.7) and Lemma 1 that the closed disk $\bar{G}_{\delta / 2}$ (i.e., the disk $|\eta| \leqq \delta / 2$ ), is mapped one-toone onto a closed neighborhood $\bar{G}_{3}, \bar{G}_{3} C G$, of $t_{0}$ in the $z$ plane in which the following holds.

LEMma 4. There exists a neighborhood $G_{3}$ of $t_{0}, \bar{G}_{3} C G$, and a function $V(z)$ such that $V(z)$ is a homeomorphic solution of

$$
V_{z}=A(z) V_{z} \text { in } G_{3}
$$

having the properties

$$
V_{z}, V_{\bar{z}} \in C_{\mu}\left(\bar{G}_{3}^{ \pm}\right) \text {and } V \in \operatorname{Lip}\left(\bar{G}_{3}\right) \text {, }
$$

where $G_{3}^{+}$and $G_{3}^{-}$are the intersections of $G_{3}$ and $G^{+}$and $G^{-}$respectively.

These are all the properties of $V(z)$ which we shall use in proving Theorem 1. Later we shall need a property of $V(z)$ which we now note. Consider the Jacobian $\left|V_{\eta}\right|^{2}-\left|V_{\bar{n}}\right|^{2}$ in $\bar{G}_{\delta}^{ \pm}$. Since $V_{\eta}$ and $V_{\bar{\eta}}$ may have jump discontinuities across $L_{\delta}$, $\left|V_{\eta}\right|^{2}-\left|V_{\bar{\eta}}\right|^{2}$ may also be discontinuous across $L_{\delta}$. From (3.26), (3.28); and (3.30)

$$
\begin{aligned}
\left|V_{\eta}\right|^{2}-\left|V_{\bar{\eta}}\right|^{2} & =\left|V_{\eta}\right|^{2}\left(1-\left|A_{\delta}^{ \pm}\right|^{2}\right) \geqq\left(1-|S(f)|^{2}\right)\left(1-\left|A_{\delta}^{ \pm}\right|^{2}\right) \\
& \geqq\left(1-10 / \mu\|f\|_{B} \delta^{\mu}\right)\left(1-\left|A_{\delta}^{ \pm}\right|^{2}\right) \text { in } \bar{G}_{\delta}^{ \pm} .
\end{aligned}
$$

From (3.29) and (3.18) we have

$$
\|f\|_{B} \leqq\left\|A_{\delta}\right\|_{B}+\left\|A_{\delta} S(f)\right\|_{B}, \quad\|f\|_{B} \leqq \frac{12 M}{1-M_{\mu}^{\prime \prime} \delta^{\mu}}
$$


Hence using (3.36)

$$
\frac{10}{\mu}\|f\|_{B} \delta^{\mu}<\frac{120 M \delta^{\mu}}{\mu\left(1-M_{\mu}^{\prime \prime} \delta^{\mu}\right)}<1
$$

and therefore from $(3.41)$

$$
\left|V_{\eta}\right|^{2}-\left|V_{\bar{\eta}}\right|^{2} \geqq M_{1}>0 \text { in } \bar{G}_{\delta}^{ \pm}
$$

for some constant $M_{1}$. Considering $z$ as a new independent variable, it is easy to see from (3.7), Lemma 1 , and (3.42) that there exists a constant $M_{2}$ such that

$$
\left|V_{z}\right|^{2}-\left|V_{\bar{z}}\right|^{2} \geqq M_{2}>0 \text { in } \bar{G}_{3}^{ \pm} .
$$

3.4. Inhomogeneous Beltrami Equation. We shall now turn our attention to equation (3.19). That is

$$
U_{\bar{\eta}}=A_{\delta}(\eta) U_{\eta}+F_{\delta}(\eta), \quad \eta \in E,
$$

where $A_{\delta}(\eta)$ and $F_{\delta}(\eta)$ are defined by (3.15) and (3.19) respectively.

We shall seek a solution of this equation of the form

$$
U(\eta)=-\frac{1}{\pi} \iint_{G_{\delta}} \frac{f(z)+F_{\delta}(z)}{z-\eta} d z d \bar{z}, \quad f \in B .
$$

We have for $\eta \in E-L_{\delta}$

$$
U_{\bar{\eta}}=f(\eta)+F_{\delta}(\eta), \quad U_{\eta}=S(f)+S\left(F_{\delta}\right) .
$$

Substituting (3.46) into (3.44) we obtain the following integral equation for $f \in B$

$$
f(\eta)-A_{\delta}(\eta) S(f)=A_{\delta}(\eta) S\left(F_{\delta}\right)
$$

Let $\delta$ satisfy (3.36). We have already shown that $A_{\delta} S(f)$ is a contraction mapping of $B$ into $B$. To prove that (3.42) has a unique solution (any solution $f \in B$ would suffice), it is sufficient to show the following lemma, the proof of which follows in a fashion similar to that of Lemma 1.2 and Lemma 1.3 and will not be given.

LeMma 5. $S\left(F_{\delta}\right)$ is continuous in $E-L_{\delta}$ and $S\left(F_{\delta}\right) \in C_{\mu}\left(\bar{G}_{\delta}^{ \pm}\right)$.

Hence from (3.25) $A_{\delta} S\left(F_{\delta}\right) \in B$ and thus by the theorem of contractive mapping there exists a unique solution $f \in B$ of (3.47). Hence $U(\eta)$ defined by (3.45), is a solution of (3.44) and in view of (3.46) and Lemmas 3 and 5, it has the properties

$$
U_{\eta}, U_{\bar{\eta}} \in C_{\mu}\left(\bar{G}_{\delta}^{ \pm}\right) \text {and } U \in \operatorname{Lip}\left(\bar{G}_{\delta}^{ \pm}\right) \text {. }
$$


Introducing $z$ as a new independent variable and again letting $G_{3}$ be as in Lemma 4, we have:

LEMMA 6. Let $G_{3}$ be as in Lemma 4. There exists a function $U(z)$ which is a solution of

$$
U_{\bar{z}}=A(z) U_{z}+F(z) \text { in } G_{3}
$$

having the properties

$$
U_{z}, U_{\bar{z}} \in C_{\mu}\left(\bar{G}_{3}^{ \pm}\right) \text {and } U \in \operatorname{Lip}\left(\bar{G}_{3}\right) .
$$

3.5. Proof of Theorem 1. The proof of Theorem 1 is based on the following lemma which is a special case of a representation theorem, due to Morrey [3], and Bers and Nirenberg [4], for elliptic equations with bounded measurable coefficients. We shall state it here in our case using the functions constructed in Lemmas 4 and 6.

LeMma 7. Let $G_{3}, A(z)$ and $F(z)$ be as above, then every generalized solution $w(z)$ of the equation

$$
w_{\bar{z}}=A(z) w_{z}+F(z)
$$

in the domain $G_{3}$ admits the representation

$$
w(z)=\phi(V(z))+U(z)
$$

where $V(z)$ and $U(z)$ are defined by Lemmas 4 and 6 respectively and $\phi$ is an analytic function in the domain $V\left(G_{3}\right)$.

We have as immediate consequence of (3.40), (3.49), and (3.51) that

$$
w_{z}, w_{\bar{z}} \in C_{\mu}\left(\bar{G}_{4}^{ \pm}\right) \text {and } w \in \operatorname{Lip}\left(\bar{G}_{4}\right),
$$

where $G_{4}$ is any disk with center at $t_{0}$ and $\bar{G}_{4} C G_{3}$.

We are now in a position to prove Theorem 1 . We first remark that in the case that $B(z) \equiv C(z) \equiv 0$ in $G$, Theorem 1 follows directly from (3.52) by taking $F(z) \equiv D(z)$ and $G_{0}=G_{4}$. Let us now consider the more general case of equation (3.1).

Let $w(z)$ be any generalized solution of (3.1) in $G_{3}$. Then from (2.11) we have that $w \in C_{\rho}\left(\bar{G}_{5}\right)$, where $\rho=(p-2) / p$ and $G_{5}$ is any disk with center at $t_{0}$ and $\bar{G}_{5} C G$. Hence in $G_{5} w(z)$ is a generalized solution of

$$
w_{\bar{z}}=A(z) w_{z}+\tilde{F}(z)
$$

where

$$
\tilde{F}(z)=B(z) w+C(z) \bar{w}+D(z),
$$

and

$$
F(z) \in C_{\rho_{1}}\left(\bar{G}_{5}^{ \pm}\right), \quad \rho_{1}=\min (\rho, \mu) .
$$


Replacing the disk $G$ by $G_{5}$ and $\mu$ by $\rho_{1}$ in Lemmas 4,6 , and 7 and in (3.52), we have that there exists a disk $G_{6}, \bar{G}_{6} C G_{5}$ in which

$$
w_{z}, w_{\tilde{z}} \in C_{\rho_{1}}\left(\bar{G}_{6}^{ \pm}\right), \quad w \in \operatorname{Lip}\left(\bar{G}_{6}\right),
$$

If $\rho_{1}=\mu \leqq \rho$ then (3.55) completes the proof of Theorem 1 if we take $G_{6}$ as $G_{0}$. For the case where $\rho_{1}=\rho<\mu$, it follows from (3.54) and (3.55) that

$$
\tilde{F}(z) \in C_{\mu}\left(\bar{G}_{6}^{ \pm}\right) \text {. }
$$

Hence replacing $G$ by $G_{6}$ in Lemmas 4,6 , and 7 and in (3.52), we have that there exists a disk $G_{0}, \bar{G}_{0} C G_{6}$, in which

$$
w_{z}, w_{\bar{z}} \in C_{\mu}\left(\bar{G}_{0}^{ \pm}\right), \quad w \in \operatorname{Lip}\left(\bar{G}_{0}\right) .
$$

which completes the proof of Theorem 1 .

4. Homeomorphic solutions of Beltrami's equation. We shall now discuss some properties of homeomorphic solutions of Beltrami's equations

$$
w_{\bar{z}}=A(z) w_{z} \text { in } G,
$$

where $A(z)$ satisfies Conditions I of $\S 3$.

THEOREM 2. Let $w(z)$ be any homeomorphic solution of (4.1) then there exists a disk $G_{0}$ with center at $t_{0}, \bar{G}_{0} C G$, such that

(i) $\left|w_{z}\right|^{2}-\left|w_{\bar{z}}\right|^{2}>0$ in $\bar{G}_{0}^{ \pm}$.

(ii) Let $t$ be any point of $\bar{L}_{0}\left(\bar{L}_{0}=L \cap \bar{G}_{0}\right)$, then

$$
w(t) \in C_{\mu}^{1}\left(\bar{L}_{0}\right), \quad d w / d s \neq 0 \quad \text { on } \bar{L}_{0},
$$

where differentiation is taken with respect to arc-length $s$ on $L$.

Proof. Let $G_{3}$ and $V(z)$ be as defined in Lemma 4. From Lemma 7 it follows that in $G_{3}$

$$
w(z)=\phi(V(z))
$$

where $\phi(V)$ is an analytic function of $V$ in the domain $V\left(G_{3}\right)$. Let $t$ be any point of $L_{3}\left(L_{3}=L \cap G_{3}\right)$, then

$$
\frac{d w}{d s}=w_{z} \frac{d t}{d s}+w_{\bar{z}} \frac{d \bar{t}}{d s}=\phi^{\prime}(V(t))\left(V_{z} \frac{d t}{d s}+V_{\bar{z}} \frac{d \bar{t}}{d s}\right)
$$

exists at all points on $L_{3}$ and where by $w_{z}, w_{\bar{z}}, V_{z}$, and $V_{\bar{z}}$ we mean the limits taken from either $G_{3}^{+}$or $G_{3}^{-}$. Let $G_{0}$ be any disk with center at $t_{0}, \bar{G}_{0} C G_{3}$. Then it follows from (3.40) and (4.5) that on $\bar{L}_{0}\left(\bar{L}_{0}=L \cap \bar{G}_{0}\right)$ that

$$
d w / d s \in C_{\mu}\left(\bar{L}_{0}\right) \text { hence } w \in C_{\mu}^{1}\left(\bar{L}_{0}\right) .
$$


Since $w(z)$ and $V(z)$ are homeomorphisms of $G_{3}$ it follows that $\phi^{\prime}(V(z)) \neq 0$ in $G_{3}$, and in view of (3.43) we have

$$
\left|w_{z}\right|^{2}-\left|w_{\bar{z}}\right|^{2}=\left|\phi^{\prime}(V(z))\right|^{2}\left(\left|V_{z}\right|^{2}-\left|V_{\bar{z}}\right|^{2}\right) \geqq J_{0}>0 \text { in } \bar{G}_{0}^{ \pm}
$$

for some constant $J_{0}$. From (4.5) and (4.7) we have for $t \in \bar{L}_{0}$

$$
|d w / d s| \geqq\left|\phi^{\prime}(V(t))\right|\left(\left|V_{z}\right|-\left|V_{\bar{z}}\right|\right)>0
$$

Statements (4.6), (4.7), and (4.8) complete the proof of Theorem 2.

5. Quasilinear equations. The results of Theorems 1 and 2 may be easily generalized to quasilinear uniformly elliptic equations of the form

$$
w_{\bar{z}}=a(z, w) w_{z}+b(z, w) .
$$

Let $t_{0}, G, G^{+}, G^{-}$, and $L$ be as before. Regarding the coefficients $a(z, w)$ and $b(z, w)$ we shall make the following assumptions.

(1) $a(z, w)$ and $b(z, w)$ satisfy a uniform Hölder condition with exponent $\mu$ on $\bar{G}^{ \pm} \times S_{k}$, where $S_{k}$ is any closed disk $|w| \leqq k$ and $k$ is arbitrary. That is, there exists a constant $M(k)$ depending only on $k$ such that for all $z_{1}$ and $z_{2} \in \bar{G}^{ \pm}$and any $w_{1}$ and $w_{2} \in S_{k}$

$$
\begin{aligned}
& \left|a^{ \pm}\left(z_{1}, w_{1}\right)-a^{ \pm}\left(z_{2}, w_{2}\right)\right| \leqq M(k)\left(\left|z_{1}-z_{2}\right|^{\mu}+\left|w_{1}-w_{2}\right|^{\mu}\right), \\
& \left|b^{ \pm}\left(z_{1}, w_{1}\right)-b^{ \pm}\left(z_{2}, w_{2}\right)\right| \leqq M(k)\left(\left|z_{1}-z_{2}\right|^{\mu}+\left|w_{1}-w_{2}\right|^{\mu}\right), \quad 0<\mu<1 .
\end{aligned}
$$

(2) Uniform ellipticity: for all $z$ in $G^{ \pm}$and all $w$

$$
|a(z, w)| \leqq a_{0}<1 .
$$

By a solution $w(z)$ of (5.1) we shall mean any function $w(z)$ having generalized first derivative (in the Sobolev sense) which belong to $L_{p}^{\text {loc }}(G)$ for some $p>2$, and which satisfies (5.1) almost everywhere in $G$.

THEOREM 3. Under conditions (5.2) and (5.3) there exists a disk $G_{0}, \bar{G}_{0} C G$, such that every solution $w(z)$ of (5.1) has the properties

$$
w_{z}, w_{\bar{z}} \in C_{\mu}\left(\bar{G}_{0}^{ \pm}\right) \text {and hence } w \in \operatorname{Lip}\left(\bar{G}_{0}\right) .
$$

Proof. Let $w(z)$ be any solution of (5.1) then, $w \in C_{\rho}\left(\bar{G}_{2}\right), \rho=(p-2) / p$, where $G_{2}$ is any disk with center at $t_{0}$ and $\bar{G}_{2} C G$. Then $|w(z)| \leqq k_{2}$ on $\bar{G}_{2}$ for some const $k_{2}$. In view of (5.2) we have for all $z_{1}, z_{2} \in \bar{G}_{2}^{ \pm}$

$$
\begin{aligned}
& \left|a^{ \pm}\left(z_{1}, w\left(z_{1}\right)\right)-a^{ \pm}\left(z_{2}, w\left(z_{2}\right)\right)\right| \leqq M_{2}\left|z_{1}-z_{2}\right|^{\rho \mu}, \\
& \left|b^{ \pm}\left(z_{1}, w\left(z_{1}\right)\right)-b^{ \pm}\left(z_{2}, w\left(z_{2}\right)\right)\right| \leqq M_{2}\left|z_{1}-z_{2}\right|^{\rho \mu}
\end{aligned}
$$


for some constant $M_{2}$ which depends only on $k_{2}, \rho, \mu$, and $C_{\rho}\left(w, \bar{G}_{2}\right)$. Hence as functions of $z$

$$
a(z, w(z)), b(z, w(z)) \in C_{\rho \mu}\left(\bar{G}_{2}\right)
$$

We have from (5.3) that $w(z)$ satisfies an elliptic equation of the form

$$
w_{\bar{z}}=a(z) w_{z}+b(z) \text { in } G_{2}
$$

where the coefficients satisfy Conditions I with $\rho \mu<\mu$ replacing $\mu$. Thus Theorem 1 is satisfied and there exists a disk $G_{1}, \bar{G}_{1} C G_{2}$, such that

$$
w(z) \in \operatorname{Lip}\left(\bar{G}_{1}\right) \text {. }
$$

In view of (5.3) we have for all $z_{1}, z_{2} \in \bar{G}_{1}^{ \pm}$,

$$
\begin{aligned}
& \left|a^{ \pm}\left(z_{1}, w\left(z_{1}\right)\right)-a^{ \pm}\left(z_{2}, w\left(z_{2}\right)\right)\right| \leqq M_{1}\left|z_{1}-z_{2}\right|^{\mu}, \\
& \left|b^{ \pm}\left(z_{1}, w\left(z_{1}\right)\right)-b^{ \pm}\left(z_{2}, w\left(z_{2}\right)\right)\right| \leqq M_{1}\left|z_{1}-z_{2}\right|^{\mu},
\end{aligned}
$$

where $M_{1}$ is a const depending only on $k$, and $\operatorname{Lip}\left(w, \bar{G}_{1}\right)$. Hence

$$
a(z, w(z)), b(z(w(z))) \in C_{\mu}\left(\bar{G}_{1}^{ \pm}\right) .
$$

Applying Theorem 1 to (5.7) in the closed disk $\bar{G}_{1}$ we have that there exists a disk $G_{0}, \bar{G}_{0} C G_{1}$, such that (5.4) holds.

THEOREM 4. Under conditions (5.2) and (5.3) there exists a disk $G_{1}, \bar{G}_{1} C G_{1}$ such that every homeomorphic solution $w(z)$ of

$$
w_{\bar{z}}=a(z, w) w_{z} \text { in } G
$$

has the properties that

(i) $\left|w_{z}\right|^{2}-\left|w_{\bar{z}}\right|^{2} \geqq J_{1}>0$ in $\bar{G}_{1}^{ \pm}$

for some constant $J_{1}$.

(ii) Let $t$ be any point of $\bar{L}_{1}, \bar{L}_{1}=L \cap \bar{G}_{1}$, then

$$
w(t) \in C_{\mu}^{1}\left(\bar{L}_{1}\right), \quad d w / d s \neq 0 \quad \text { on } \bar{L}_{1} .
$$

Proof. From Theorem 3 we have that $w \in \operatorname{Lip}\left(\bar{G}_{0}\right)$ for some appropriately chosen disk $\bar{G}_{0} C G$. Hence $a(z, w(z)) \in C_{\mu}\left(\bar{G}_{0}\right)$ and the conditions of Theorem 2 are satisfied with $G_{0}$ replacing $G$. Let $G_{1}, \bar{G}_{1} C G_{0}$, be any disk for which (4.3) and (4.4) hold and hence (5.12) and (5.13) follow. 


\section{APPENDIX}

Proof of Lemma 3. Let $G_{2 \delta}$ be a disk of radius $2 \delta$ with center at the origin and $G_{2 \delta}^{+}$and $G_{2 \delta}^{-}$be the corresponding parts of $G_{2 \delta}$ which lie in the upper half and lower half planes respectively. Then since $f \equiv 0$ outside $G_{\delta}^{+}$

$$
\begin{aligned}
S(f) & =-\frac{1}{\pi} \iint_{G_{\delta}} \frac{f(z)}{(z-\eta)^{2}} d z d \bar{z}=-\frac{1}{\pi} \iint_{G_{2 \delta}} \frac{f(z)}{(z-\eta)^{2}} d z d \bar{z} \\
& =-\frac{1}{\pi} \iint_{G_{2 \delta}^{+}} \frac{f(z)}{(z-\eta)^{2}} d z d \bar{z}-\frac{1}{\pi} \iint_{G_{\overline{2}}^{-}} \frac{f(z)}{(z-\eta)^{2}} d z d \bar{z} \\
& =g_{1}(\eta)+g_{2}(\eta), \quad \eta \in G_{\delta}^{ \pm} .
\end{aligned}
$$

We note that

$$
C\left(f, \bar{G}_{2 \delta}^{ \pm}\right)=C\left(f, \bar{G}_{\delta}^{ \pm}\right), \quad H\left(f, \bar{G}_{2 \delta}^{ \pm}, \mu\right)=H\left(f, \bar{G}_{\delta}^{ \pm}, \mu\right) .
$$

Denote by $g_{1}^{+}(\eta), g_{1}^{-}(\eta)\left(g_{2}^{+}(\eta), g_{2}^{-}(\eta)\right)$ the values of $g_{1}(\eta)\left(g_{2}(\eta)\right)$ for $\eta \in G_{\delta}^{+}$and $G_{\delta}^{-}$respectively. In what follows we shall estimate the moduli and Hölder constants of $g_{1}^{+}(\eta)$ and $g_{1}^{-}(\eta)$. It can be easily seen that the same estimates may be obtained for $g_{2}^{-}(\eta)$ and $g_{2}^{+}(\eta)$ respectively. Many of the integral estimates we shall need are classical and may be found in Vekua [1], if such is the case we shall merely say that the estimate may be obtained in a standard manner. Consider first $g_{1}^{+}(\eta)$.

$$
\begin{aligned}
g_{1}^{+}(\eta) & =-\frac{1}{\pi} \iint_{G_{2 \delta}^{+}} \frac{f(z)-f(\eta)}{(z-\eta)^{2}}-f(\eta) \phi_{\dot{G}_{2 \delta}^{+}}^{\prime}(\eta)=I_{1}+I_{2}, \\
\phi_{\dot{G}_{2 \delta}^{+}} & =\frac{1}{2 \pi i} \int_{\dot{G}_{2 \delta}^{+}} \frac{\bar{z} d z}{(z-\eta)}, \quad \phi_{\dot{G}_{2 \delta}^{+}}^{\prime}(\eta)=\frac{1}{2 \pi i} \int_{\dot{G}_{2 \delta}^{+}} \frac{\bar{z} d z}{(z-\eta)^{2}} .
\end{aligned}
$$

Splitting the boundary integral into two parts it can be easily seen that

$$
\begin{aligned}
\phi_{\dot{G}_{2 \delta}^{+}}^{\prime}(\eta) & =\frac{1}{2 \pi i} \int_{-2 \delta}^{2 \delta} \frac{x d x}{(x-\eta)^{2}}+\frac{2}{\pi} \int_{0}^{\pi} \frac{\delta^{2}}{\left(2 \delta e^{i \theta}-\eta\right)^{2}} d \theta \\
& =\frac{1}{2 \pi i}\left(\log \frac{\eta-2 \delta}{\eta+2 \delta}+\frac{4 \delta \eta}{\left(\eta^{2}-4 \delta^{2}\right)}+4 i \int_{0}^{\pi} \frac{\delta^{2}}{\left(2 \delta e^{i \theta}-\eta\right)^{2}} d \theta\right),
\end{aligned}
$$

where we take any single valued branch of the log cut from $-2 \delta$ to $2 \delta$ along the real axis. For $\eta \in G_{\delta}^{+}$or $G_{\delta}^{-}$we have $\delta \leqq|\eta \pm 2 \delta| \leqq 3 \delta$ and using (A.5), a computation shows that

$$
\left|\phi_{\dot{G}_{2 \delta}^{+}}^{\prime}(\eta)\right| \leqq 4 \quad \text { for } \eta \in G_{\delta}^{ \pm}
$$

Using (A.6) and (3.24) in $I_{2}$ and a standard estimate for $I_{1}$ in (A.3) we find for $\left|g_{1}^{+}(\eta)\right|$ and in a similar manner for $\left|g_{2}^{-}(\eta)\right|$ that since $0<\mu<1$,

$$
\left|g_{1}^{+}(\eta)\right| \leqq \frac{10}{\mu} H\left(f, \bar{G}_{\delta}^{+}, \mu\right) \delta^{\mu}, \quad\left|g_{2}^{-}(\eta)\right| \leqq \frac{10}{\mu} H\left(f, \bar{G}_{\delta}^{-}, \mu\right) \delta^{\mu}
$$


Consider $g_{1}^{-}(\eta)$ which is an analytic function of $\eta$. A separation of $g_{1}^{-}(\eta)$ into two parts as is (A.3) will not easily work since $f(\eta)$ is in general discontinuous across $L_{\delta}$ and $f(z)-f(\eta)$ does not satisfy a Hölder condition. This however may be overcome by noticing that for $\eta \in G^{-}, \bar{\eta}$ belongs to $G_{\delta}^{+}$and for any $z \in G_{2 \delta}^{+}$

$$
|z-\bar{\eta}| \leqq|z-\eta| \text {. }
$$

Then since $0<\mu<1$,

$$
g_{1}^{-}(\eta)=-\frac{1}{\pi} \iint_{G_{2 \delta}^{+}} \frac{f(z)-f(\bar{\eta})}{(z-\eta)^{2}} d z d \bar{z}-f(\bar{\eta}) \phi_{\dot{G}_{2 \delta}^{+}}^{\prime}(\eta)=I_{3}+I_{4},
$$

$$
\left|I_{3}\right| \leqq \frac{H\left(f, \bar{G}_{\delta}^{+}, \mu\right)}{\pi} \iint_{G_{2 \delta}^{+}} \frac{|z-\bar{\eta}|^{\mu}}{|z-\bar{\eta}|^{2}} d z d \bar{z} \leqq \frac{6}{\mu} H\left(f, \bar{G}_{\delta}^{+}, \mu\right) \delta^{\mu} .
$$

Using (A.10), (A.6), and (3.24) in (A.9) we obtain

$$
\left|g_{1}^{-}(\eta)\right| \leqq \frac{10}{\mu} H\left(f, \bar{G}_{\delta}^{+}, \mu\right) \delta^{\mu}, \quad\left|g_{2}^{+}(\eta)\right| \leqq \frac{10}{\mu} H\left(f, \bar{G}_{\delta}^{-}, \mu\right) \delta^{\mu},
$$

where the estimate for $\left|g_{2}^{+}(\eta)\right|$ may be obtained in a similar fashion. Combining (A.7) and (A.11) we obtain

$$
|S(f)| \leqq \frac{10}{\mu}\left(H\left(f, \bar{G}_{\delta}^{+}, \mu\right)+H\left(f, \bar{G}_{\delta}^{-}, \mu\right)\right) \delta^{\mu} \quad \text { for } \eta \in G_{\delta}^{ \pm} .
$$

We shall now estimate the Hölder continuity of $S(f)$ in $G_{\delta}^{+}$and $G_{\delta}^{-}$. Let $\eta_{1}$, $\eta_{2} \in G_{\delta}^{+}$then

$$
\begin{aligned}
g_{1}^{+}\left(\eta_{1}\right)-g_{1}^{+}\left(\eta_{2}\right)= & \frac{\left(\eta_{1}-\eta_{2}\right)}{\pi} \iint_{G_{2 \delta}^{+}} \frac{f(z)-f\left(\eta_{1}\right)}{\left(z-\eta_{2}\right)\left(z-\eta_{1}\right)^{2}} d z d \bar{z} \\
& +\frac{\left(\eta_{1}-\eta_{2}\right)}{\pi} \iint_{G_{2 \delta}^{+}} \frac{f(z)-f\left(\eta_{2}\right)}{\left(z-\eta_{2}\right)^{2}\left(z-\eta_{1}\right)} d z d \bar{z} \\
& +\left(f\left(\eta_{2}\right)-f\left(\eta_{1}\right)\right)\left(\frac{\bar{\eta}_{2}-\bar{\eta}_{1}}{\eta_{1}-\eta_{2}}+\frac{\phi_{\dot{G}_{2 \delta}^{+}}\left(\eta_{1}\right)-\phi_{\dot{G}_{2 \delta}^{+}}\left(\eta_{2}\right)}{\eta_{1}-\eta_{2}}+\phi_{\dot{G}_{2 \delta}^{+}}^{\prime}\left(\eta_{2}\right)\right) \\
& +f\left(\eta_{1}\right)\left(\phi_{\dot{G}_{20}^{+}}^{\prime}\left(\eta_{1}\right)-\phi_{\dot{G}_{2 \delta}^{+}}^{\prime}\left(\eta_{2}\right)\right)=I_{5}+I_{6}+I_{7}+I_{8} .
\end{aligned}
$$

$I_{5}$ and $I_{6}$ may be estimated in a standard way. It can be shown that

$$
\left|I_{5}\right|,\left|I_{6}\right| \leqq M_{\mu}^{\prime} H\left(f, \bar{G}_{\delta}^{+}, \mu\right)\left|\eta_{1}-\eta_{2}\right|^{\mu},
$$

where $M_{\mu}^{\prime}$ is a constant which depends only on $\mu, 0<\mu<1$, and in particular is independent of $\delta$. It follows from (A.6) that

$$
\left|I_{7}\right| \leqq 9 H\left(f, \bar{G}_{\delta}^{+}, \mu\right)\left|\eta_{1}-\eta_{2}\right|^{\mu}
$$

and a computation using (A.5) shows that

$$
\left|\phi_{\dot{G}_{2 \delta}^{+}}^{\prime}\left(\eta_{1}\right)-\phi_{\dot{G}_{2 \delta}^{+}}^{\prime}\left(\eta_{2}\right)\right| \leqq \frac{16}{\delta^{\mu}}\left|\eta_{1}-\eta_{2}\right|^{\mu}
$$


for any $\eta_{1}, \eta_{2}$ both belonging to either $G^{+}$or $G^{-}$. Using (A.16) and (3.24) we obtain

$$
\left|I_{8}\right| \leqq 16 H\left(f, \bar{G}_{\delta}^{+}, \mu\right)\left|\eta_{1}-\eta_{2}\right|^{\mu} .
$$

Combining (A.14), (A.15), and (A.17) we have

$$
\left|g_{1}^{+}\left(\eta_{1}\right)-g_{1}^{+}\left(\eta_{2}\right)\right| \leqq\left(2 M_{\mu}^{\prime}+25\right) H\left(f, \bar{G}_{\delta}^{+}, \mu\right)\left|\eta_{1}-\eta_{2}\right|^{\mu}
$$

and in a similar fashion one can obtain

$$
\left|g_{2}^{-}\left(\eta_{1}\right)-g_{2}^{-}\left(\eta_{2}\right)\right| \leqq\left(2 M_{\mu}^{\prime}+25\right) H\left(f, \bar{G}_{\delta}^{+}, \mu\right)\left|\eta_{1}-\eta_{2}\right|^{\mu} .
$$

We shall now estimate the Hölder continuity of the analytic function $g_{1}^{-}(\eta)$. This again can be easily done by using the device previously used. Namely if $\eta_{1}, \eta_{2} \in G_{\delta}^{-}$then $\bar{\eta}_{1}, \bar{\eta}_{2} \in G_{\delta}^{+}$and it can be easily shown that

$$
\begin{aligned}
g_{1}^{-}\left(\eta_{1}\right)-g_{1}^{-}\left(\eta_{2}\right)= & \frac{\left(\eta_{1}-\eta_{2}\right)}{\pi} \iint_{G_{2 \delta}^{+}} \frac{f(z)-f\left(\bar{\eta}_{1}\right)}{\left(z-\eta_{2}\right)\left(z-\eta_{1}\right)^{2}} d z d \bar{z} \\
& +\frac{\left(\eta_{1}-\eta_{2}\right)}{\pi} \iint \frac{f(z)-f\left(\bar{\eta}_{2}\right)}{\left(z-\eta_{2}\right)^{2}\left(z-\eta_{1}\right)} d z d \bar{z} \\
& +\left(f\left(\bar{\eta}_{2}\right)-f\left(\bar{\eta}_{1}\right)\right)\left(\frac{\phi_{\dot{G}_{20}^{+}}^{+}\left(\eta_{1}\right)-\phi_{\dot{G}_{20}^{+}}^{+}\left(\eta_{2}\right)}{\eta_{1}-\eta_{2}}-\phi_{\dot{G}_{2 \delta}^{+}}^{\prime}\left(\eta_{2}\right)\right) \\
& +f\left(\bar{\eta}_{1}\right)\left(\phi_{\dot{G}_{20}^{+}}^{\prime}\left(\eta_{1}\right)-\phi_{\dot{G}_{20}^{+}}\left(\eta_{2}\right)\right)=I_{9}+I_{10}+I_{11}+I_{12} .
\end{aligned}
$$

For $z \in G_{2 \delta}^{+}$the inequalities $\left|z-\bar{\eta}_{1}\right| \leqq\left|z-\eta_{1}\right|$ and $\left|z-\bar{\eta}_{2}\right| \leqq\left|z-\eta_{2}\right|$ hold and therefore $I_{9}$ and $I_{10}$ may be estimated by

$$
\begin{gathered}
\left|I_{9}\right| \leqq \frac{\left|\eta_{1}-\eta_{2}\right|}{\pi} H\left(f, \bar{G}_{\delta}^{+}, \mu\right) \iint_{G_{2 \delta}^{+}} \frac{d z d \bar{z}}{\left|z-\eta_{2}\right|\left|z-\eta_{1}\right|^{2-\mu}} \\
\leqq M_{\mu}^{\prime} H\left(f, \bar{G}_{\delta}^{+}, \mu\right)\left|\eta_{1}-\eta_{2}\right|^{\mu}, \\
\quad\left|I_{10}\right| \leqq M_{\mu}^{\prime} H\left(f, \bar{G}_{\delta}^{+}, \mu\right)\left|\eta_{1}-\eta_{2}\right|^{\mu},
\end{gathered}
$$

where $M_{\mu}^{\prime}$ is the same as in (A.14).

The estimates for $I_{11}$ and $I_{12}$ proceed in the same manner as those for $I_{7}$ and $I_{8}$ and we obtain

(A.23) $\left|I_{11}\right| \leqq 8 H\left(f, \bar{G}_{\delta}^{+}, \mu\right)\left|\eta_{1}-\eta_{2}\right|^{\mu}, \quad\left|I_{12}\right| \leqq 16 H\left(f, \bar{G}_{\delta}^{+}, \mu\right)\left|\eta_{1}-\eta_{2}\right|^{\mu}$.

Combining (A.21), (A.22), and (A.23) we have

$$
\left|g_{1}^{-}\left(\eta_{1}\right)-g_{1}^{-}\left(\eta_{2}\right)\right| \leqq\left(2 M_{\mu}^{\prime}+25\right) H\left(f, \bar{G}_{\delta}^{+}, \mu\right)\left|\eta_{1}-\eta_{2}\right|^{\mu},
$$

and in a similar fashion

$$
\left|g_{2}^{+}\left(\eta_{1}\right)-g_{2}^{+}\left(\eta_{2}\right)\right| \leqq\left(2 M_{\mu}^{\prime}+25\right) H\left(f, \bar{G}_{\delta}^{-}, \mu\right)\left|\eta_{1}-\eta_{2}\right|^{\mu} .
$$


Combining (A.18) and (A.25), (A.19) and (A.24) respectively we have

$$
H\left(S(f), G_{\delta}^{ \pm}, \mu\right) \leqq\left(2 M_{\mu}^{\prime}+25\right)\left(H\left(f, \bar{G}_{\delta}^{+}, \mu\right)+H\left(f, \bar{G}_{\delta}^{-}, \mu\right)\right) .
$$

Since the estimates (A.12) and (A.26) are independent of the distance to the boundaries $\dot{G}_{\delta}^{+}$and $\dot{G}_{\delta}^{-}$it follows that $S(f) \in C_{\mu}\left(\bar{G}_{\delta}^{ \pm}\right)$and

$$
C\left(S(f), \bar{G}_{\delta}^{ \pm}\right) \leqq \frac{10}{\mu}\|f\|_{B}, \quad H\left(S(f), \bar{G}_{\delta}^{ \pm}, \mu\right) \leqq\left(2 M_{\mu}^{\prime}+25\right)\|f\|_{B},
$$

which completes the proof of the lemma.

\section{BIBLIOGRAPHY}

1. I. N. Vekua, Generalized analytic functions, Fizmatgiz, Moscow, 1959; English transl., Addison-Wesley, Reading, Mass., 1962.

2. E. Cumberbatch, L. Sarason and H. Weitzner, Magnetohydrodynamic flow past a thin airfoil, AIAA. J. 1 (1963), 679-690.

3. C. B. Morrey, On the solution of quasi-linear elliptic partial differential equations, Trans. Amer. Math. Soc. 43 (1938), 126-166.

4. L. Bers and L. Nirenberg, On a representation theorem for linear elliptic systems with discontinuous coefficients and its applications, Convegno Internazionale, sulle Equazioni Lineari alle Derivate Parziali, pp. 111-140, Edizioni Cremonese, Rome, 1955.

5. L. Bers, F. John and M. Schecter, Partial differential equations, Interscience, New York, $1964 ;$ p. 138.

6. N. I. Mushelišvilli, Singular integral equations, Noordhoff, Groningen, 1953. (Translated from Russian.)

7. L. Lichtenstein, Zur theorie der konformen abbildung konforme, Bull. Acad. Sci. Cracovie Ser. A (1916), 192-217.

8. F. Riesz and B. Sz.-Nagy, Functional analysis, Ungar, New York, 1955. (Translated from French, Leçons d'analyse fonctionelle, 2nd ed., Akademiai Kiadó, Budapest, 1953.)

9. B. Bojarski, Homeomorphic solutions of Beltrami systems, Dokl. Akad. Nauk SSSR 102 (1955), 661-664.

Cornell University, ITHACA, New York 